\section{Clinical history}

We report the case of a 53 years-old women followed in our institution for a refractory thyroid carcinoma. She had a past history of a left breast carcinoma treated with surgery, chemotherapy and radiotherapy in 1992. The story begins in 2007 , when a follicular thyroid carcinoma pT2NXMX was discovered and treated with surgery and $100 \mathrm{mCi}$ of radioactive iodine. 3 years later, she was diagnosed refractory thyroid carcinoma because of a high thyroglobulin assay $(477 \mathrm{ug} / \mathrm{l})$ without uptake on post-therapeutic (131I) whole body scan. Thereafter, the story is marked by the appearance in 2011 of multiple cervical and mediastinal lymph nodes, pulmonary and bones lesions. For metastatic bone disease, she underwent several surgeries: a right T5 pediculectomy with T3-T7 osteosynthesis, metastasectomy for a right parietal lesion of the skull and for a left humeral lesion. She underwent an additive radiation therapy for vertebral and left humeral lesions. She had also benefited from a cementoplasty for a right ischial tuberosity lesion in 2012 . Zoledronic acid was given regularly to prevent skeletal-related events. In parallel, we observed pulmonaries lesions progression with an aspect of lymphangitic carcinomatosis. In early 2013, morphologic evaluation showed the appearance of multiple lymph nodes (hepatic hilum, inguinal bilaterally). Clinically, she described a chronic dry cough with a shortness of breath. Biologically, we noted a slight increase of thyroglobulin assay $(711 \mathrm{ug} / \mathrm{l})$.
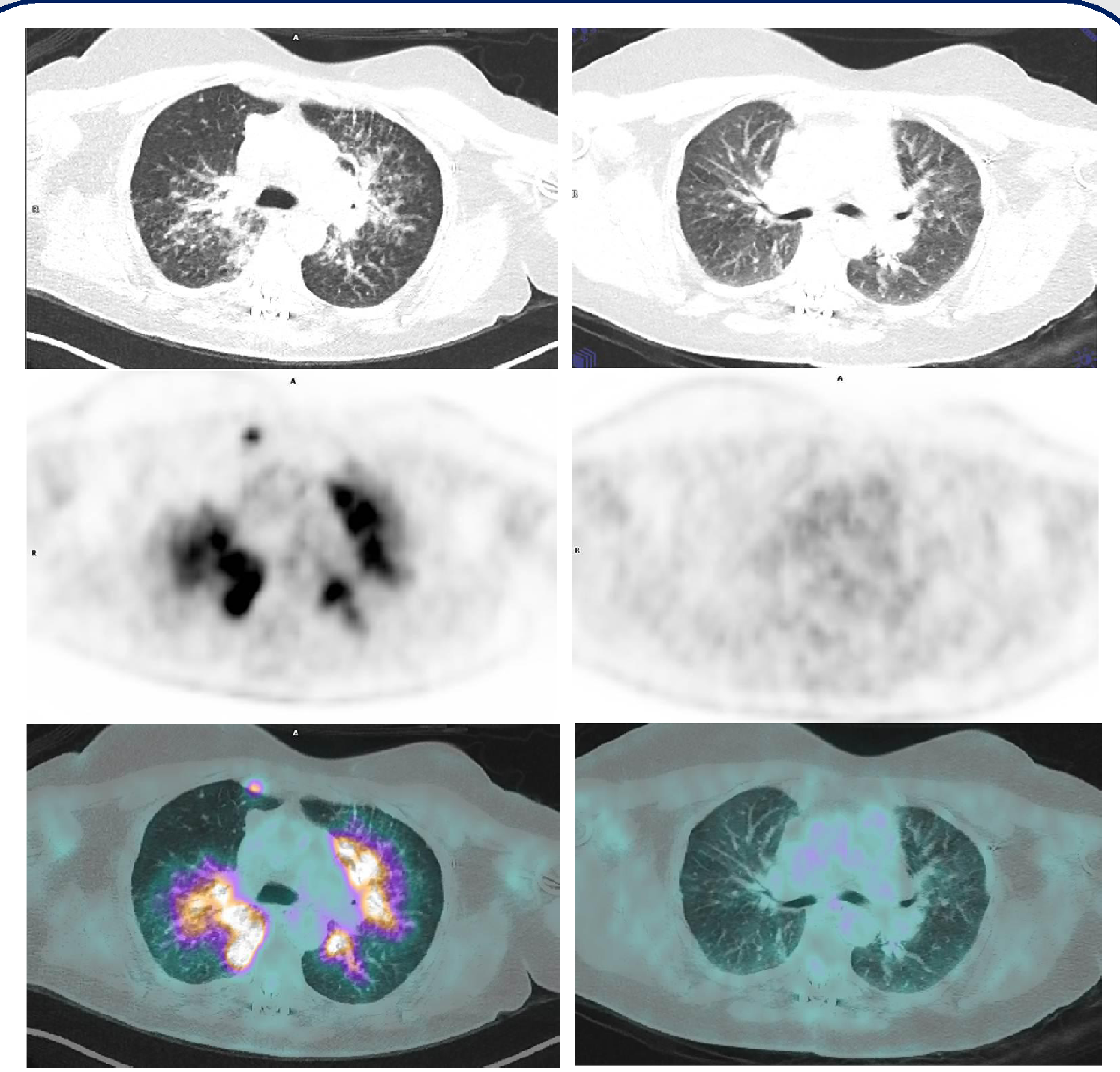

Figure 2: Images for our 53-year-old patient with bilateral pulmonary lesions : CT scan image (A), FDG scan (B) and coregistered PET/CT scan before (left) and 4 months after corticosteroids (right)

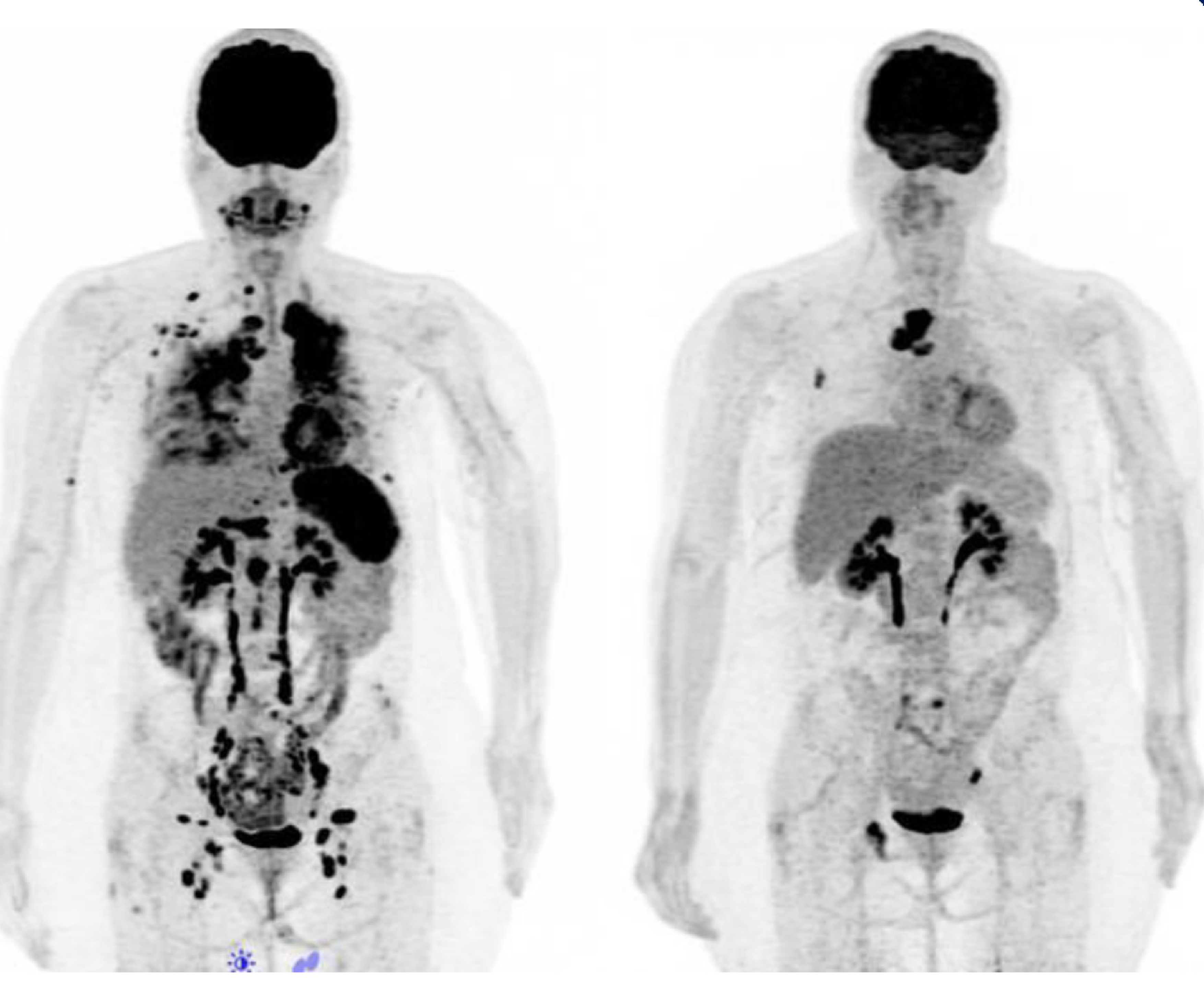

Figure 1: Images for our 53-year-old patient : maximum intensity projection of FDG-PET before (left) and 4 months after corticosteroids (right)

\section{Therapeutic intervention and outcome}

In front of this supposed disease progression and after agreement of the TUTHYREF network, we introduced, in may 2013, a tyrosin kinase inhibitor, Sorafenib $400 \mathrm{mg}$ twice a day. After six months of treatment, a therapeutic evaluation was performed. Clinically, the patient described a worsening respiratory status. TEP-TDM showed a majoration of mediastinal, abdominal and inguinal lymph nodes and pulmonary lesions uptakes (SUV max 12,8). TEP-TDM showed also appearance of an intense splenic uptake. Biologically, thyroglobulin assay remained paradoxically stable $(673 \mathrm{ug} / \mathrm{l})$,

Because of this atypical presentation for a thyroid metastasis disease (lymphangitic carcinomatosis, unusual splenic metastasis, stable thyroglobulin assay) and past history of breast cancer, a biopsy of a left inguinal lymph node was finally performed. Histological analysis demonstrated an aspect of epitheloid cell granuloma reaction with no caseous necrosis. This aspect was consistent with the diagnosis of sarcoidosis. Angiotensin-converting enzyme was slightly increased $(95,7$ U/L [20-70]),

Therapeutically, Sorafenib was discontinued and we started a corticotherapy, prednisone $60 \mathrm{mg}$ once a day in november 2013, enabling an improvement of respiratory symptoms. TEP-TDM performed in April 2014 showed a disappearance of all lymph nodes, pulmonaries and splenic uptakes (fig. 1 and fig. 2)

\section{Conclusion}

Sarcoïdosis is a relatively frequent systemic disease of unknown origin. Prevalence is about 4,7 - 64 in 100000 [1]. In our case, regression of pulmonaries, lymph nodes and splenic lesions, without other treatment than corticosteroids, have confirmed their belonging to sarcoïdosis. Sarcoidosis mimicking recurrences cancer has already been described [2],

Diagnosis of sarcoidosis should be considered in case of atypical metastatic disease, when presentation is consistent with this diagnosis, especially in case of discover of FDG-avid lesions discovered during follow-up [3]

\section{Bibliography}

1. Valeyre and al. Lancet. 2014; 383(9923):1155-67

2. Cormio and al, J Obstet Gynaecol 13 nov 2014;1-2

3. Zivin and al, Internal Medicine 2014;53(21):2555-6 